\title{
Educación-Acción: Herramientas para Afrontar la Injusticia Ambiental en una Comunidad Costera
}

\section{Action-Education: Tools to Address Environmental Injustice in a Coastal Community}

\author{
Ana M. Raimondo ${ }^{1 *}$ \\ F. Javier Perales-Palacios ${ }^{2}$ \\ José Gutiérrez-Pérez ${ }^{2}$ \\ Susana Vidoz ${ }^{1}$ \\ ${ }^{1}$ Universidad Nacional de la Patagonia San Juan Bosco, Argentina \\ ${ }^{2}$ Universidad de Granada, España
}

\begin{abstract}
Este artículo pretende implementar un proceso de concienciación, empoderamiento y compromiso ciudadano. Se identifica el caso objeto de atención, un barrio costero de la ciudad de Comodoro-Rivadavia (Argentina) afectado por un problema de contaminación en el que se interviene con un programa de voluntariado universitario. Se realiza un estudio de caso a través de una estrategia de investigación-acción; la muestra la constituyen voluntarios universitarios, vecinos y responsables institucionales; los instrumentos de obtención de datos son de corte esencialmente cualitativo. Los resultados muestran que la acción educativa llevada a cabo consigue movilizar a la comunidad vecinal, haciéndole tomar conciencia del problema y generando en ella un empoderamiento que conduce a diferentes acciones que van desde la denuncia y la confrontación hasta la recreación de lazos comunitarios. En el programa la justicia social y la justicia ambiental tienden puentes que permiten pensar el futuro desde la toma de conciencia ciudadana de las condiciones de contaminación de su territorio, las causas y consecuencias ligadas a aspectos en los que lo político se entrecruza con los intereses económicos y la supervivencia en condiciones extremas de explotación de recursos naturales.
\end{abstract}

Descriptores: Justicia social; Voluntariado; Contaminación; Sensibilización ambiental; Autonomización.

This article aims to implement a process of citizen awareness, empowerment and commitment. The case is identified: a coastal area of the city of Comodoro-Rivadavia (Argentina) affected by a problem of pollution in which a university volunteer program is involved. A case study through an action-research strategy is used; the sample is constituted by university volunteers, neighbours and institutional leaders; the instruments of obtaining data are essentially qualitative. The results show that the educational action carried out can mobilize the neighbourhood community, making them aware of the problems and generating in it an empowerment that leads to different actions ranging from denunciation and confrontation to the recreation of community ties. In the program, social justice and environmental justice have bridges that allow us to think about the future from the citizen's awareness of the pollution conditions of its territory, the causes and consequences linked to aspects in which the political intersects with economic interests and survival in extreme conditions of exploitation of natural resources.

Keywords: Social justice; Volunteers; Pollution; Environmental awareness; Empowerment.

*Contacto: ana.raimondo@gmail.com

ISSN: 2254-3139

www.rinace.net/riejs/

revistas.uam.es/riejs
Recibido: $\quad 19$ de mayo 2017

$1^{\text {a }}$ Evaluación: 25 de octubre 2017

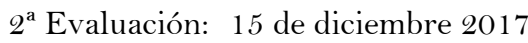

Aceptado: 15 de enero 2018 


\section{Introducción}

La justicia social y la justicia ambiental constituyen sendos discursos que consolidan valores social y moralmente deseables en nuestro mundo actual, y que abren nuevas perspectivas al análisis de la realidad socioambiental en la que nos movemos. Vincularlos constituye una oportunidad para enfatizar los estrechos lazos entre ambos conceptos de justicia y la necesidad de abordarlos globalmente.

La educación -desde su aspiración emancipatoria- constituye el eslabón adecuado e imprescindible para enlazar estos dos campos, entendidos tanto como horizonte de apertura y contingencia de la acción social como herramientas operativas que permiten llevar a cabo procesos rigurosos de análisis crítico de la realidad.

La educación -desde una perspectiva normativa e instrumental- permite construir compromisos consensuados de intervención sistemática y evaluación de logros, que efectivamente comporten avances significativos en la calidad de vida de las comunidades humanas y en la dignificación de sus condiciones de existencia, incluyendo lo ambiental como derecho fundamental del ser humano. En este artículo describimos un estudio de caso localizado en un barrio costero argentino afectado por un problema crónico de contaminación, y en el que una acción educativa llevada a cabo por voluntarios universitarios consigue movilizar a la comunidad vecinal, haciéndole tomar conciencia del problema y generando en ella un empoderamiento que moviliza diferentes acciones que van desde la denuncia y la confrontación hasta la recreación de lazos comunitarios.

\section{Fundamentación teórica}

En el contexto de la educación científica los conceptos de justicia social y de justicia ambiental han ido ganando protagonismo en la literatura educativa durante los últimos años (Maulucci, 2012).

Con relación a la justicia social, se han señalado como algunas de sus prioridades el cómo mejorar el rendimiento académico de grupos de estudiantes marginados (Rodríguez, 1997). Más concretamente, Maulucci (2012) señala la tipología de discursos y las ideas centrales que amparan la investigación sobre el tópico de justicia social en el marco de la educación científica (cuadro 1).

Existen escasos precedentes que afronten experiencias para promover ambos tópicos. Señalemos el trabajo de Paige y Hardy (2014), en el que utilizan controversias sociocientíficas para construir el currículo de ciencias y matemáticas de los futuros profesores y "ayudar mejor a sus estudiantes a entender cómo se puede vivir y actuar de una forma más social en un mundo ecológicamente justo" (p. 18). Asimismo, Mateos, Bejarano, Franco y Moreno (2014) trabajaron con alumnos de Educación Infantil los valores de igualdad de género, los recursos naturales y la equidad, y la pobreza mediante el cuento y el juego de simulación.

En cuanto a la justicia ambiental, la noción de ambiente que postula el ambientalismo hegemónico (hijo del discurso colonial euro-americano sobre la naturaleza), con su carácter esencialista (en tanto muestra la naturaleza como un espacio edénico, en el que conviven armónica y equilibradamente las especies con el hombre) ha sido cuestionada, apostando por otra noción de ambiente que re-significa la relación entre la naturaleza y la comunidad. De esta manera, la justicia ambiental entiende el ambiente como el lugar 
(geográfico, cultural y emocional) donde se desarrolla la vida cotidiana de las personas, mediado por los discursos y prácticas de las distintas comunidades que históricamente lo han habitado, dando forma a la tierra y a los bienes comunales.

Cuadro 1. Fundamentos de la investigación sobre justicia social en la educación científica

\begin{tabular}{|c|c|}
\hline DISCURSO & IDEAS CENTRALES \\
\hline Feminista & $\begin{array}{l}\text { Insta a repensar la naturaleza de la ciencia y la educación científica. } \\
\text { Propone una educación científica liberadora en lugar de opresiva. } \\
\text { Posiciona el conocimiento como subjetivo y contextualmente mediado. } \\
\text { Se aleja de los programas compensatorios. }\end{array}$ \\
\hline Multicultural & $\begin{array}{l}\text { Nociones de los desafíos de la ciencia basadas en la tradición occidental. } \\
\text { Insta al uso de una pedagogía culturalmente relevante y receptiva y } \\
\text { ciencia para el yo y la transformación social. } \\
\text { Enfatiza el papel de la acción comunitaria. }\end{array}$ \\
\hline Crítico & $\begin{array}{l}\text { Critica el papel de las escuelas y las instituciones en la reproducción de la } \\
\text { inequidad. } \\
\text { Destaca el papel de la hegemonía, el poder y el privilegio en } \\
\text { sostener la opresión. } \\
\text { Critica la enculturación y la reproducción de la cultura dominante. } \\
\text { Luchas para abordar las desigualdades arraigadas. }\end{array}$ \\
\hline $\begin{array}{l}\text { Ciencia para } \\
\text { todos }\end{array}$ & $\begin{array}{l}\text { Posiciona la alfabetización científica como un objetivo nacional. } \\
\text { Afirma objetivos de equidad en la educación científica. } \\
\text { Aclara la naturaleza de la ciencia. } \\
\text { Hace hincapié en los métodos basados en la investigación para la } \\
\text { enseñanza y el aprendizaje de las ciencias. }\end{array}$ \\
\hline
\end{tabular}

Fuente: Recuperada de Maulucci (2012, p. 587), traducción realizada por los autores.

Reconociendo pues diversas concepciones del ambiente, desde la justicia ambiental se pueden cuestionar las desigualdades (sociales y ambientales) encubiertas por el ambientalismo hegemónico relativas a las condiciones materiales de vida y a la invisibilidad social efectiva que padecen los grupos más vulnerables y marginados. El hiperconsumo y la sobreexplotación que producen los grupos más poderosos contaminan y destruyen principalmente el ambiente que habitan los sectores más vulnerables.

Al respecto es interesante incluir los pensamientos de Latapí (1995) cuando expresa:
Las injusticias de las sociedades latinoamericanas no son un destino fatal, sus causas -políticas, económicas y culturales-podrían superarse en relativamente poco tiempo si se conjuntaran acciones políticas decididas, reclamos populares organizados y procesos sociales - como el de la educación-impulsados con energía. Nunca la justicia ha sido un don, sino una conquista. (...) los hechos, a lo largo de las décadas, patentizan la ineficacia de los esfuerzos por disminuir sustancialmente las desigualdades; en los últimos años, inclusive, comprueban su incremento; ellos permiten calificar de 'máscaras de la justicia' las soluciones hasta ahora intentadas. (p. 3)

La justicia ambiental, definida como un espacio tanto de activismo social como de producción académica, en el cual se integran las demandas de salud, vivienda, educación, trabajo, asociadas al bienestar social con las demandas y reclamos ante las diversas formas de contaminación y degradación del ambiente, ha ido enriqueciendo el discurso de la Educación para la Sostenibilidad en la medida que acentúa la dimensión social de la misma y proclama la necesidad de ir caminando en paralelo a la justicia social. En este sentido se orientaron bastantes de las acciones promovidas por la Década UNESCO de la Educación para el Desarrollo Sostenible (2004-2014): 
La Década de la Educación para el Desarrollo Sostenible se propuso impulsar una educación solidaria que contribuya a una correcta percepción del estado del mundo, que sea capaz de generar actitudes y compromisos responsables, y que prepare a los ciudadanos para una toma de decisiones fundamentadas dirigidas al logro de un desarrollo culturalmente plural, socialmente justo y ecológicamente sostenible, que supere las posiciones antropocéntricas clásicas y que esté orientada a la búsqueda de modelos más comprensivos e inteligentes de interacción $n^{t}$.

La justicia ambiental concibe la soberanía como una "agencia productiva" que crea normas y procedimientos que definen el ejercicio del poder (Carrizo, 2012). Un poder inspirado en premisas de democracia deliberativa y dialógica, construido sobre la problematización continuada de las comunidades y sus intereses vitales, tales como el uso racional y equitativo de los recursos naturales o la ocupación del territorio frente a agresiones al mismo como la explotación abusiva de recursos. Y esa soberanía no se regala, se construye desde la base de la continua reivindicación y lucha por el establecimiento de cotas de justicia y equidad de alto alcance. A tal fin la justicia ambiental debería promover el reconocimiento de identidades individuales y colectivas para la construcción de innovadoras formas institucionales para la inclusión y participación efectivas.

En la centenaria ciudad de Comodoro Rivadavia (Argentina) los efectos del histórico déficit de infraestructura de servicios y de planificación urbana (Vázquez, 2015) impactan desigualmente sobre los distintos grupos sociales en función de su lugar de residencia, raza/etnia, lugar de origen y capital social disponible. De ahí que la noción de justicia ambiental permita articular toda esta serie de privaciones de derechos que describimos en este artículo. Las ideas y prácticas sobre la comunidad son el resultado de la relación entre el lugar geográfico, cultural y emocional, donde humanos y el ambiente convergen en interacciones problematizadoras en las que la educación aporta acción vertebradora de soberanía. Concebir a la naturaleza como comunidad, implica reconocer la interdependencia dada entre diversidad de formas sociales, culturales y emocionales que coexisten en la especie humana y de otras especies, y su necesaria coexistencia como "unidad en la diferencia".

De esta manera, los supuestos de la equidad en la distribución del riesgo ambiental, el reconocimiento de la diversidad de los participantes o experiencias en comunidades afectadas y participación en los procesos políticos que crean y administran políticas ambientales, hacen de la justicia ambiental una categoría teórica y práctica con destacado carácter performativo (Schlosberg, 2004). La potencialidad de la noción de justicia ambiental parte entonces de poder articular ideas y prácticas de ambiente como comunidad (pluridiversa), que puede alumbrar una soberanía popular, basada en un poder asociativo, en la que puedan tener lugar novedosas y diversas instituciones inclusivas y participativas para la efectiva toma de decisión colectiva.

La idea de justicia ambiental engarza de lleno con toda la tradición del pensamiento proactivo de Paolo Freire (1970) y con sus postulados de pedagogía problematizadora cuando expresa que:

Quanto mais se problematizam os educandos, como seres no mundo e com o mundo, tanto mais se sentirão desafiados. Tão mais desafiados, quanto mais obrigados a responder ao desafio Desafiados, compreendem o desafio na própria ação de captá-lo. Mas, precisamente porque captam o deafio como um problema em suas conexões com

\footnotetext{
${ }^{1}$ www.oei.es/decada/acciono04.htm
} 
outros, num plano de totalidade e nao como algo petrificado, a compreensão resultante tende a tornar-se crescentemente crítica, por isto, cada vez mais desalienada. (p. 40)

Esta idea de problematización de Freire entendida como acción colectiva de reflexión coordinada sobre lo que se dice y lo que se hace, buscando el porqué de las cosas, o el para qué de ellas, constituye una base esencial de la propuesta de intervención en el barrio Stella Maris donde se desarrolla nuestro proyecto. Problematizar es la base de la solución para resolver las condiciones de injusticia social en sus diferentes planos de realidad en nuestra comunidad. La problematización nace de la conciencia que los ciudadanos adquieren al darse cuenta de que saben poco de sí, de que desconocen elementos esenciales de su territorio y de las interacciones locales y globales que se producen en él; y de que ese desconocimiento contribuye a que esos problemas sean insolubles siendo ellos los mayores afectados en la merma de su soberanía.

En cuanto al voluntariado y las motivaciones que lo rigen han sido ya ampliamente estudiados (Liarakou, Kostelou y Gavrilakis, 2011). Entre las evidencias más relevantes que ha puesto de manifiesto la literatura disponible destaca que el trabajo por proyectos ambientales mejora los conocimientos y actitudes de los estudiantes participantes (AlBalushia y Al-Aamri, 2014), estos hallazgos justifican nuestro modelo de investigaciónacción orientado al trabajo por proyectos con universitarios.

El objetivo principal de este trabajo es mostrar y describir un caso de intervención social que pretende, a través de un programa de voluntariado universitario, despertar entre los habitantes de una zona ambientalmente degradada la conciencia de la situación en que viven y, en último término, promover su movilización en aras de cambiar esas condiciones sobrevenidas. Las dos cuestiones que han guiado esta investigación han sido las siguientes:

- ¿Cuáles son las dimensiones de la vulnerabilidad global que hacen que los pobladores costeros del barrio Stella Maris sean vulnerables frente a los peligros identificados?

- ¿Pueden establecerse escenarios frente a dichos peligros, tanto de la comunidad como de los que toman las decisiones?

\section{Método}

\subsection{Contexto}

\subsubsection{La zona costera de Comodoro Rivadavia}

El caso de estudio se sitúa en un barrio costero del municipio de Comodoro Rivadavia. Se trata de una costa de condiciones naturales relativamente difíciles para la operatividad portuaria y donde las geoformas marinas se suman a condiciones climáticas asiduamente adversas, para ofrecer también un marco natural poco amigable. De cualquier modo, está visto que se trata de condicionamientos y no de limitantes, como lo prueban, entre otros ejemplos, las adecuaciones técnicas en materia de maniobras para la carga del crudo (Raimondo, 2008), al tratarse de una zona petrolífera.

A los efectos de la ordenación y gestión local de la zona costera comodorense, el foco de atención lo centramos en una delgada franja que se extiende desde la línea de alta marea promedio hasta la primera línea de edificación costera, ya que es allí donde acontecen los efectos más relevantes de la interacción entre los subsistemas físico-natural, socioeconómico y jurídico-administrativo (Barragán, 2003; Raimondo 2010). Sorensen, Mc 
Creary y Brandani (1992) destacan que la característica distintiva de la zona litoral es la coexistencia de ambientes costeros, recursos costeros y usos costeros; de aquí que lo que defina a la zona sea la agregación de distintos sistemas, que para Barragán (2003) son sistemas abiertos, complejos en su estructura, extremadamente dinámicos y con dificultad de predicción de las repercusiones de la intervención humana.

Trascendiendo a los subsistemas considerados por Barragán (2003), se sitúa la esfera del conocimiento o gnosfera, que es mencionada desde hace décadas por diferentes autores, entre ellos George (1972) o Bunge (1989), quienes abordan la temática ambiental desde una perspectiva holística y en un escenario de fuerzas equivalentes entre las denominadas ciencias "duras" y "blandas". Gnosfera es aquella parcela del conocimiento humano en donde convergen todas las ideas, conceptos y sistema de valores que cada persona recibe y asimila como tales, y que por medio de su voluntad puede cambiar la estructura de la realidad. Dentro de la gnosfera destacamos su componente educativa, al considerar que solo desde ese nivel se modificarán los modos de abordaje de los escenarios de estudio constituidos por la mutua interacción sociedad-naturaleza, en este caso, el escenario costero (figura 1).

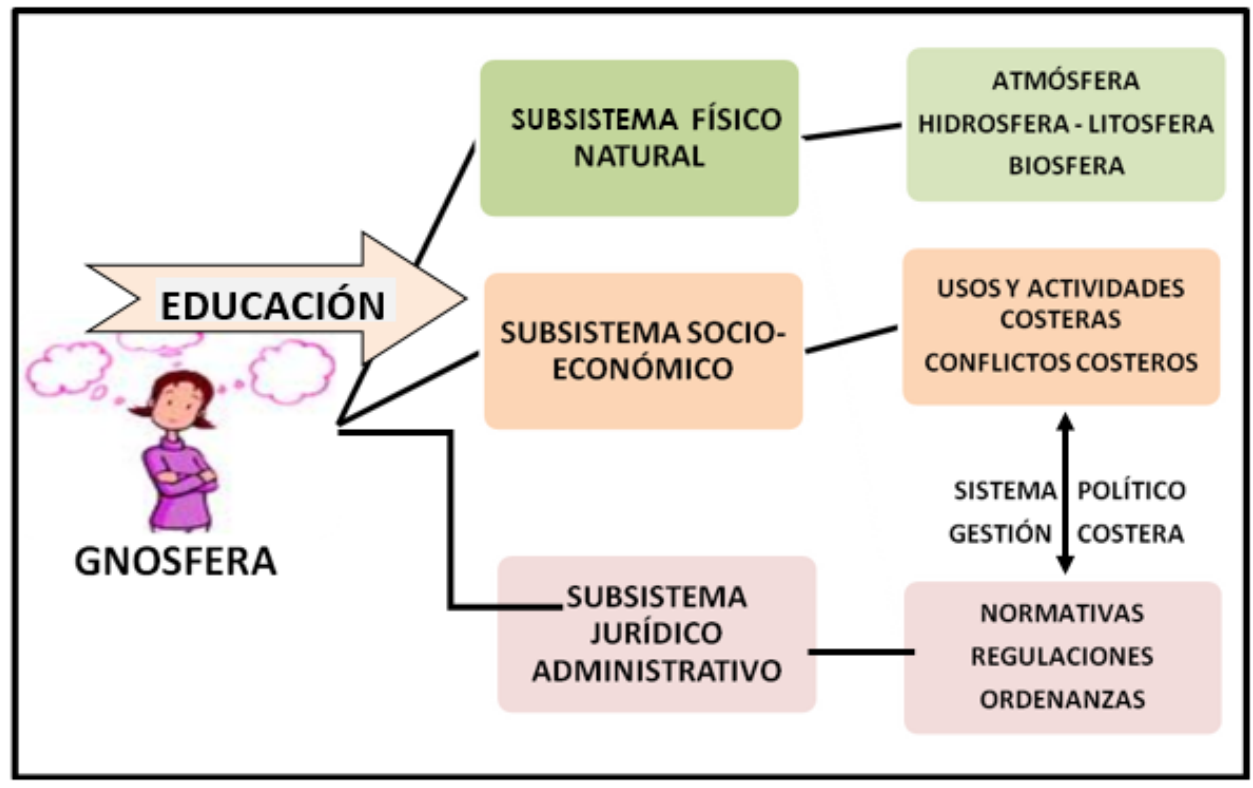

Figura 1. Sistema de conocimiento aplicado al espacio litoral Fuente: Elaboración propia.

Un recorrido a lo largo de la costa comodorense permitirá reconocer oleoductos, cañerías abandonadas, pasarelas, pozos petroleros inactivos, vestigios de antiguos vertidos y otros pasivos ambientales de la actividad petrolera, que generan una apariencia de "abandono", lo que permite afirmar que la costa pareciera haberse destinado al uso predominante de "patio trasero" de la ciudad. A ello hay que añadir rieles y durmientes abandonados o el basural de la ciudad, modificando un espacio de belleza escénica invalorable. Por otra parte, un importante número de efluentes cloacales y pluviales desembocan a lo largo de la línea de costa, desaguando en el mar, en el mejor de los casos sólo con tratamiento primario, provocando no solo el impacto visual que es el más evidente, sino también el deterioro ambiental general del área. 


\subsubsection{El barrio de Stella Maris}

Este barrio se encuentra ubicado en el subsistema que concentra los usos y actividades más desfavorables, y si bien estas condiciones de deterioro costero son un común denominador en todos los subsistemas determinados, alcanzan en este sitio su máxima expresión, siendo el sector costero de mayor nivel de degradación ambiental de la ciudad. Basurales clandestinos, vertidos, olores y humos de la industria frigorífica, efluentes cloacales y pluviales determinan un paisaje de alto grado de deterioro. Resulta paradójico que, en un pasado no tan lejano, que al decir de los vecinos refiere a las décadas de los 60 y 70 del siglo Xx, este sector era utilizado como balneario debido al predominio de arenas, la extensión de la playa y a su orientación. Se la denominaba la playa del 99, haciendo alusión al pozo No 99 de la ex empresa estatal YPF. En la actualidad, el deterioro ambiental y el nivel de contaminación de las aguas lo inhabilitan para tal fin, por lo cual existe cartelería indicando la prohibición de uso balneario, aunque muchos vecinos la utilizan para actividades de tiempo libre, recolección de moluscos y pesca recreativa.

En este sector predominan los usos urbanos como lo constituyen las escuelas de nivel inicial, primario y secundario, iglesia, comercios, centro de promoción barrial y el centro de salud. Las viviendas en general muestran un cierto estado de precariedad. En los últimos años la expansión del frente urbano en este sitio obedece a asentamientos espontáneos sobre terrenos que ya han llegado a ocupar predios muy próximos al basural municipal.

\subsection{El estudio de caso mediante investigación-acción}

Según Hitchcock y Hughes (1995, en Cohen, Manion y Morrinson, 2005) los estudios de caso:

(a) se establecen en contextos temporales, geográficos, organizativos, institucionales y de otro tipo que permiten delimitar el caso;(b) pueden definirse con referencia a las características determinadas por los individuos y grupos involucrados; $y(c)$ pueden definirse por los roles y funciones de los participantes en el caso. (p. 182)

Como tipología, dentro de un estudio de caso, este puede considerarse como una observación participante ya que los investigadores forman parte activa del proceso, que se inserta en el marco de una estrategia de investigación-acción. La investigación-acción es un concepto que involucra no solamente un modo de aproximarse a un objeto de investigación sino también una postura explícita del investigador unido a su objeto.

Las investigaciones enmarcadas en la investigación-acción transcurren a lo largo de un continuo en el que emergen tres racionalidades diferentes: la técnica, la práctica y la crítica o emancipatoria (Zuber-Skerritt, 1996, en Suárez-Pazos, 2002:4). La investigación-acción crítica incorpora los objetivos de las otras perspectivas, pero le añade la finalidad de emancipación de los participantes que hasta el momento solo han sido protagonistas de un modelo coercitivo y jerárquico en la toma de las decisiones. El rol del investigador que se involucra en esta corriente es el de alguien que asume el compromiso de compartir con los participantes la experiencia de la autogestión de sus problemáticas en una postura que, en palabras de Freire (1970), se define como activista y militante, luchando por un contexto más justo y democrático por medio de la reflexión crítica de las ideas y de las conductas. De este modo las comunidades se enrolan en acciones de prudencia y de precaución ya que habrá muchas fuerzas de tipo coercitivo a las que se tendrán que enfrentar. 
Por lo tanto, si pretendemos inscribir nuestra tarea dentro de la investigación-acción crítica tendremos un doble compromiso ético con la información que generemos: el del investigador en sí mismo, pero también con los datos que hemos obtenido de esa comunidad en la que se centra nuestra tarea, ya que dicha investigación dará cuenta no solo del contexto del colectivo estudiado, sino además de las propias interpretaciones de quien lo describe y analiza.

Este tipo de investigación está orientada al cambio y la transformación de la realidad, cuestiona los principios de una cultura extractivista y economicista e intenta reforzar la cultura de pertenencia, de compromiso, de resistencia y de solidaridad. Se construye en la interacción con el medio (la casa, el hábitat urbano, la aldea, la biorregión, etc.) y se vincula a la tierra, al agua, a los paisajes y al resto de seres vivos, forjando la vida compartida en y por la pluralidad de los lugares en los que vivimos sucesiva o alternativamente a lo largo de nuestras vidas, otrora más sedentarias y hoy más nómadas (Sauvé, 2006, p. 94).

La educación ambiental tiene un papel esencial en la construcción de esta identidad, desde la convicción de que nuestra identidad psicosocial y cultural está tejida con nuestra misma identidad ecológica (Carvalho, 2004). Y en este itinerario, la educación ambiental se convierte en el instrumento esencial a través de la investigación-acción para integrar la crítica y el análisis social en el corazón de los problemas ambientales y de los patrones de justicia que han de regularlos, donde el cuestionamiento crítico deviene en un bumerán que nos vuelve a traer aquí, ahora y entre nosotros más allá de la denuncia, marcándonos el camino y clarificando qué podemos hacer dentro de una visión global y holística de esa realidad.

\subsection{El programa de intervención}

El programa comienza con una acción formativa destinada a los alumnos universitarios de la Universidad Nacional de la Patagonia San Juan Bosco, quienes, una vez cumplida, enfrentaron el desafío de encarar su trabajo de educación ambiental en un ámbito no formal y de cruda realidad con una problemática ambiental real (Raimondo, Monti, Perales y Gutiérrez, 2017). Dicho programa tiene una diversidad de estrategias: por un lado, la formación a lo largo de un cuatrimestre dentro de la Cátedra de Educación Ambiental en la cual se analizan los marcos teóricos asociados al Paradigma de la Complejidad, la Ética Ambiental y las herramientas de intervención tales como las técnicas alternativas de resolución de conflictos, juegos ambientales, simulacros de foros, ejercicios de roles, entre otros. Culmina con la realización de proyectos de Educación Ambiental que se destinan a diferentes colectivos de los barrios alcanzados por el voluntariado. Además, se realiza la formación específica en temáticas puntuales, a saber: definición de actores sociales, conflictos, participación ciudadana, vulnerabilidad, riesgo, peligrosidad, sinergia, entre otros.

El programa de intervención sigue las pautas exigidas por la Secretaría de Políticas universitarias dependiente del Ministerio de Educación. En la misma se exige el compromiso de acciones conjuntas entre las instituciones de los barrios. En nuestro caso se realizaron convenios con las escuelas de nivel inicial, primario y secundario, la unión vecinal, el centro de promoción barrial y el centro de salud. Para iniciar la tarea, durante 2011 se realizaron entrevistas en profundidad a los habitantes de las 106 viviendas que componen las manzanas costeras del barrio. Aquellas proporcionaron datos fundamentales para el diagnóstico socioambiental a partir del cual poder determinar indicadores de vulnerabilidad educativa e institucional, resistencia y resiliencia. Este 
diagnóstico nos posibilitó observar el interés común por mejorar las condiciones de calidad ambiental del sitio en que se desenvuelven. Tras el programa formativo se llevaron a cabo diversas actividades con la comunidad objeto de atención y que incluyeron:

- Salidas de campo con la comunidad para identificar problemáticas.

- Realización de talleres internos de capacitación entre los voluntarios y las instituciones involucradas.

- Elaboración de listas de chequeo ambiental.

- Elaboración de material audiovisual (videos con vecinos y videos en la playa)

- Elaboración de folletos, materiales divulgativos y de difusión en colaboración con la comunidad. Confección de murales.

- Generación de cartografía temática socio-ambiental a partir de las herramientas SIG.

- Certámenes destinados a los jóvenes con la propuesta de recuperar la historia ambiental del barrio.

- Promoción de actividades de reacondicionamiento de un Parque (juegos, fogones, espacio verde, etc.)

- Evaluación continua de las acciones propuestas y evaluación final del proyecto. Generación de informes parciales y del informe final.

\subsection{Muestra}

Para el caso de esta investigación la muestra está constituida por los vecinos de las manzanas costeras del barrio Stella Maris. El barrio es mucho más amplio, pero se seleccionó únicamente el sector de las manzanas más próximas a la costa atendiendo también al hecho de que se trata de la zona que ocuparon en la década de 1950 los primitivos habitantes del sector antes de que existiera urbanización alguna. Otra parte de la muestra está conformada por los representantes de las instituciones de base que gestionan directamente el territorio cotidiano.

Por último, se consideró a los voluntarios, alumnos y docentes del ámbito universitario, como otro de los componentes de la muestra al ser ellos quienes vienen desarrollando y supervisando las tareas de educación ambiental en la zona de interés de manera ininterrumpida desde hace más de diez años. De este modo queda determinada la población que se sintetiza en el cuadro 2.

\subsection{Instrumentos de recogida de información}

De un total de 106 viviendas que componen las manzanas costeras del barrio, pudieron concretarse 60 entrevistas (56,5\%). En un $20 \%$ de los casos (21 viviendas), los vecinos se negaron a ser entrevistados y en un $23,5 \%$ (25 viviendas) no se hallaron presentes sus moradores en el domicilio o se trataba de viviendas, en apariencia, deshabitadas.

Durante el proceso se consideró relevante la información de base que podría ofrecernos la comunidad vecinal debido a que es ella quien guarda la experiencia de lo cotidiano. También lo fueron las vivencias de los integrantes del voluntariado y alumnos universitarios que desarrollaron tareas particulares con distintos actores. Por su parte, los referentes del barrio, en especial los que hace varios años que están a cargo de las 
instituciones, serían los que podrían proporcionar, a través de sus relatos y participación activa, una recopilación del espacio vivido volcado en palabras.

Cuadro 2. Muestra correspondiente a la comunidad costera $(\mathrm{N}=60)$

\begin{tabular}{lr}
\hline & $\mathbf{N}$ \\
\hline Hombres mayores de 65 & 7 \\
Mujeres mayores de 65 & 7 \\
Hombres entre 35 a 65 & 14 \\
Mujeres entre 35 a 65 & 14 \\
Hombres menores de 35 & 11 \\
Mujeres menores de 35 & 7 \\
\hline
\end{tabular}

Fuente: Elaboración propia.

En términos estadísticos se llevó a cabo un muestreo no probabilístico, también llamado intencional o de conveniencia, ya que hubo una intencionalidad de obtener muestras representativas, incluyendo a las personas que "a priori" creíamos que reunirían las características prediseñadas a los fines de la investigación. También se procedió a utilizar la técnica denominada "bola de nieve" mediante la cual al iniciar el proceso un individuo va conduciendo a otro y este a otro hasta conseguir una muestra que resulte suficiente (Hernández-Sampieri, Fernández y Baptista, 1991). Los diferentes instrumentos empleados se describen a continuación en función de la muestra diana de agentes que han participado aportando información en cada una de las fases del proyecto de intervención: vecinos, voluntarios universitarios y agentes institucionales (cuadros 3 y 4 ).

\subsubsection{De los vecinos de las manzanas costeras del barrio}

A partir de un guion de entrevista diseñado a esos efectos, y previamente probado para definir si las preguntas eran válidas y suficientes para obtener los datos de base que necesitaríamos, se inicia la capacitación de los alumnos universitarios para poder encarar el trabajo de campo con los vecinos del barrio. La base de este trabajo de campo se sustenta en la realización de entrevistas.

Aquellas constaban de dieciséis preguntas básicas y tanto el guion como el entrenamiento se realizaron pensando en conceptos teóricos previos y teniendo presentes los siguientes intereses:

- Recuperar datos muy generales sobre la historia de la familia en el barrio, la composición de su grupo familiar y su relación con el mundo del trabajo (contexto).

- Obtener respuestas que llevaran a entender cómo perciben su medio ambiente costero.

- Que pudieran indicar alguna pista sobre su vulnerabilidad educativa.

- Que recuperaran la opinión sobre la tarea de aquellos que toman decisiones en la política ambiental de su barrio costero. Ello a su vez permitiría generar una lista de actores que los vecinos costeros reconocen en el barrio y algunas pistas respecto de la vulnerabilidad institucional.

- Que indiquen su opinión respecto de quiénes son los responsables del estado ambiental de su costa. Ello a su vez podría referir indirectamente a los aspectos de resistencia y resiliencia.

- Establecer posibles relaciones entre las condiciones del ambiente y la salud. 
Cuadro 3. Muestra correspondiente a las instituciones de base territorial

\begin{tabular}{lcccc}
\hline & N & CUESTIONARIOS & TALLERES & ENTREVISTAS \\
\hline Presidente de la UV* & 1 & $\mathrm{X}$ & $\mathrm{X}$ & $\mathrm{X}$ \\
Secretaria de la UV & 1 & $\mathrm{X}$ & $\mathrm{X}$ & $\mathrm{X}$ \\
Directora escuela primaria & 1 & $\mathrm{X}$ & $\mathrm{X}$ & $\mathrm{X}$ \\
Director colegio secundario & 1 & $\mathrm{X}$ & $\mathrm{X}$ & $\mathrm{X}$ \\
Director cultura MCR $*$ & 1 & $\mathrm{X}$ & $\mathrm{X}$ & $\mathrm{NP}$ \\
Directora hábitat MCR & 1 & $\mathrm{X}$ & $\mathrm{X}$ & $\mathrm{X}$ \\
Subsecretario de ambiente MCR & 3 & $\mathrm{X}$ & $\mathrm{X}$ & $\mathrm{X}$ \\
ONG APO & 1 & $\mathrm{X}$ & $\mathrm{X}$ & $\mathrm{X}$ \\
Ministerio de Ambiente del Chubut & 2 & $\mathrm{X}$ & $\mathrm{NP}$ & $\mathrm{NP}$ \\
Club de ciencias & 1 & $\mathrm{X}$ & $\mathrm{NP}$ & $\mathrm{X}$ \\
Autoridades universitarias & 2 & & $\mathrm{X}$ & $\mathrm{NP}$ \\
Técnicos consultados & 2 & $\mathrm{X}$ & $\mathrm{X}$ & $\mathrm{X}$ \\
\hline
\end{tabular}

Nota: Total de la muestra $=17 . \mathrm{X}$ : participación o utilización de las herramientas de consulta. NP: no procede. * Unión vecinal. ** Subsecretaría de Medio ambiente.

Fuente: Elaboración propia.

Cuadro 4. Muestra correspondiente a los actores universitarios

\begin{tabular}{|c|c|c|c|c|c|c|}
\hline & $\mathbf{N}$ & CAPACITACIÓN & TALLERES & SENDEROS & CUESTIONARIOS & PEA* \\
\hline Vol. alumnos & 21 & $\mathrm{X}$ & $\mathrm{X}$ & $\mathrm{X}$ & $\mathrm{X}$ & \\
\hline Vol. docentes & 7 & $\mathrm{X}$ & $\mathrm{X}$ & & $\mathrm{X}$ & \\
\hline $\begin{array}{l}\text { Alumnos de ed. } \\
\text { ambiental } \\
2011 / 12 / 13\end{array}$ & 45 & $\mathrm{X}$ & $\mathrm{X}$ & $\mathrm{X}$ & & $\mathrm{X}$ \\
\hline
\end{tabular}

Nota: Total de la muestra $=73$. * PEA: Proyectos de Educación Ambiental. X: participación o utilización de las herramientas de consulta.

Fuente: Elaboración propia.

También se procuró identificar el lote y la manzana correspondiente con la idea de valorar si existía algún tipo de asociación entre el sitio que habita la persona entrevistada y las respuestas obtenidas (Raimondo, Gutiérrez y Perales, 2012).

El guion, que constituyó el protocolo para la entrevista en campo se utilizó únicamente como referencia, ya que se pretendía establecer una conversación franca y abierta con el entrevistado; tenía la característica de repetir de una u otra manera un concepto de forma que se tuviera asegurada la obtención del dato. El promedio de tiempo de cada entrevista fue de unos veinte minutos. Ello dependía del número de adultos interesados en responder en cada vivienda.

El resultado de cada entrevista se volcó luego en un informe final por manzana tratando, en todos los casos, de diferenciar de entre las respuestas las que se basan en datos rastreables de aquellas que aparecían como opiniones personales sustentadas en su propio imaginario o intuición. Si bien estas últimas no se desecharon, se tomó nota sobre las respuestas del tipo "a mí me parece... oyó creería que..." Posteriormente se volcaron los datos en matrices. 


\subsubsection{De los voluntarios universitarios}

Los voluntarios fueron esenciales a la hora del trabajo de intervención. La mayoría de ellos pertenecen a la Carrera de Licenciatura en Gestión Ambiental, otros al Profesorado de Geografía, al de Historia y a la Tecnicatura en Sistemas de Información Geográfica. Son los responsables de dirigir las actividades a diferentes colectivos del barrio, desde obras de teatro de títeres hasta dinamización de Talleres con vecinos adultos. La mayoría de ellos se encuentran cursando el tercer año de su carrera universitaria de grado.

A efectos de poder recuperar algunas de las experiencias que se obtuvieron con el trabajo voluntario en el barrio Stella Maris, se diseñó una encuesta de autoevaluación que fue enviada vía e-mail a cada uno de los participantes de los proyectos de voluntariado, tanto a los alumnos como a los docentes que lo integran. La opción de realizar una encuesta en lugar de una entrevista fue dejar en libertad de acción a los voluntarios para responderla si esa era su opción. Su porcentaje de respuesta indicaría una prueba de compromiso, además permitiría ponderar los aspectos relacionados a los marcos teóricos trabajados durante estos años y su nivel de apropiación.

La finalidad primordial de la encuesta fue la de evaluar los aspectos formativos de la herramienta del voluntariado a lo largo de un proceso de intervención comunitaria desarrollado desde hacía tres años en el barrio. El formato constó básicamente de tres aspectos: el primero referido al relato de la propia experiencia individual a partir del cual pudimos obtener las vivencias; el segundo se relaciona con los aprendizajes y productos obtenidos de la experiencia de voluntariado y el planteo de la posibilidad de darle continuidad a las acciones desplegadas en el territorio; y el tercero centrado en la percepción sobre los efectos del voluntariado tanto en el trabajo de campo como en los propios voluntarios. Sobre veintiocho participantes que integraron los tres grupos de voluntariados, respondieron dieciocho miembros: cinco docentes, un graduado y doce alumnos.

\subsubsection{De los referentes institucionales}

Del mismo modo que se hizo con los docentes y alumnos integrantes del voluntariado, se procedió a enviar una encuesta a aquellos colectivos que tuvieron relación directa con las acciones de educación ambiental desplegadas en el territorio, o que por su rol institucional o profesional pudieran evaluar el alcance de las propuestas de educación ambiental en el barrio Stella Maris. A estos efectos se envió el cuestionario a los representantes de las instituciones del barrio y a las autoridades de la Facultad de Humanidades. También se realizaron dos encuestas de control aprovechando la visita a la ciudad de profesionales españoles en el año 2012, quienes interactuaron en dos actividades de educación ambiental. Una de ellas fue el recorrido para elaborar senderos de interpretación en la costa y Playa del 99 y otra fue la asistenciaal cierre de uno de los proyectos que se desarrolló en el Colegio Secundario del barrio Stella Maris. En varios casos quedaron casilleros vacíos o sin respuestas ya que, al ser generales, las preguntas en ocasiones no aplicaban al caso particular de consulta.

\footnotetext{
${ }^{2}$ Los voluntariados universitarios fueron financiados por el Ministerio de Educación de la Nación a través de la Secretaría de Políticas Universitarias (SPU) en los años 201 1, 2012 y 2013. Los dos primeros destinados al barrio costero Stella Maris y el ultimo para el barrio Restinga Ali ambos de la ciudad de Comodoro Rivadavia.
} 
Se reunieron un total de trece encuestas respondidas y las preguntas que integraron la encuesta fueron muy similares a las que realizamos a los voluntarios, pero para este caso, en vez de estar centradas en el voluntariado se tuvieron en cuenta la totalidad de las herramientas de educación ambiental desplegadas. En la encuesta enviada se solicitaron los datos referidos a cuántos años consideraban que nos encontrábamos trabajando con actividades de educación ambiental en el barrio, ya que esa información nos permitiría medir la dimensión temporal de la percepción de estos actores sobre nuestra intervención.

También se les solicitó un relato escrito desde la mirada de su institución que permitiera obtener sus percepciones de las experiencias de educación ambiental en áreas costeras desarrolladas. Se indagó respecto a los productos obtenidos, la referencia a los problemas ambientales del barrio y el grado de involucramiento personal e institucional con las mismas. Se indagó igualmente respecto de su opinión sobre la continuidad o no de estas actividades de educación ambiental con preguntas de opciones de valoración entre Nada, Algo, Bastante, Mucho y Totalmente, respecto de las consecuencias de dichas actividades.

Toda la información recogida de las encuestas se volcó en tablas Excel que permitieron el análisis y las síntesis de categorías de forma inductiva mediante un análisis del contenido (Raimondo, 2014).

\section{Resultados}

En este apartado se irán contextualizando e interpretando los datos obtenidos con los distintos instrumentos utilizados en torno a los interrogantes de investigación, continuando con un epígrafe relativo al papel desempeñado por la educación ambiental. Finalmente organizaremos las principales ideas en un DAFO (Debilidades, Amenazas, Fortalezas, Oportunidades) integrado que ha permitido dar continuidad al trabajo y seguir interviniendo en el barrio mejorando los ingredientes del programa de intervención socioeducativa dinamizado desde la cátedra de educación ambiental de la Universidad Nacional de la Patagonia San Juan Bosco de Comodoro y optimizando los niveles de implicación de los universitarios.

Las dos cuestiones iniciales que han servido de referencia para la discusión y análisis interpretativo de la información recabada son las siguientes:

- ¿Cuáles son las dimensiones de la vulnerabilidad global que hacen que los pobladores costeros del barrio Stella Maris sean vulnerables frente a los peligros identificados?

- ¿Pueden establecerse escenarios frente a dichos peligros, tanto de la comunidad como de los que toman las decisiones?

\subsection{La vulnerabilidad global de los habitantes. Estrategia basada en la educación ambiental}

La estrategia que nos propusimos consistió en identificar de manera participativa cuáles eran los eventos peligrosos frente a los cuales la comunidad del barrio Stella Maris se sentía vulnerable. Adhiriendo a los aportes de Prades-López y González-Reyes (1999) es preciso evaluar la peligrosidad, tanto aquella socialmente percibida como técnicamente evaluada, a fin de establecer cuál es en definitiva la fuente de origen de dicha peligrosidad. Los talleres realizados con la comunidad que hemos denominado "Tarde de Playón, Recuperando la memoria ambiental de la playa y Educación ambiental formal", fueron 
dando pautas de cuáles son para la comunidad las principales fuentes de peligro de contaminación (figura 2).
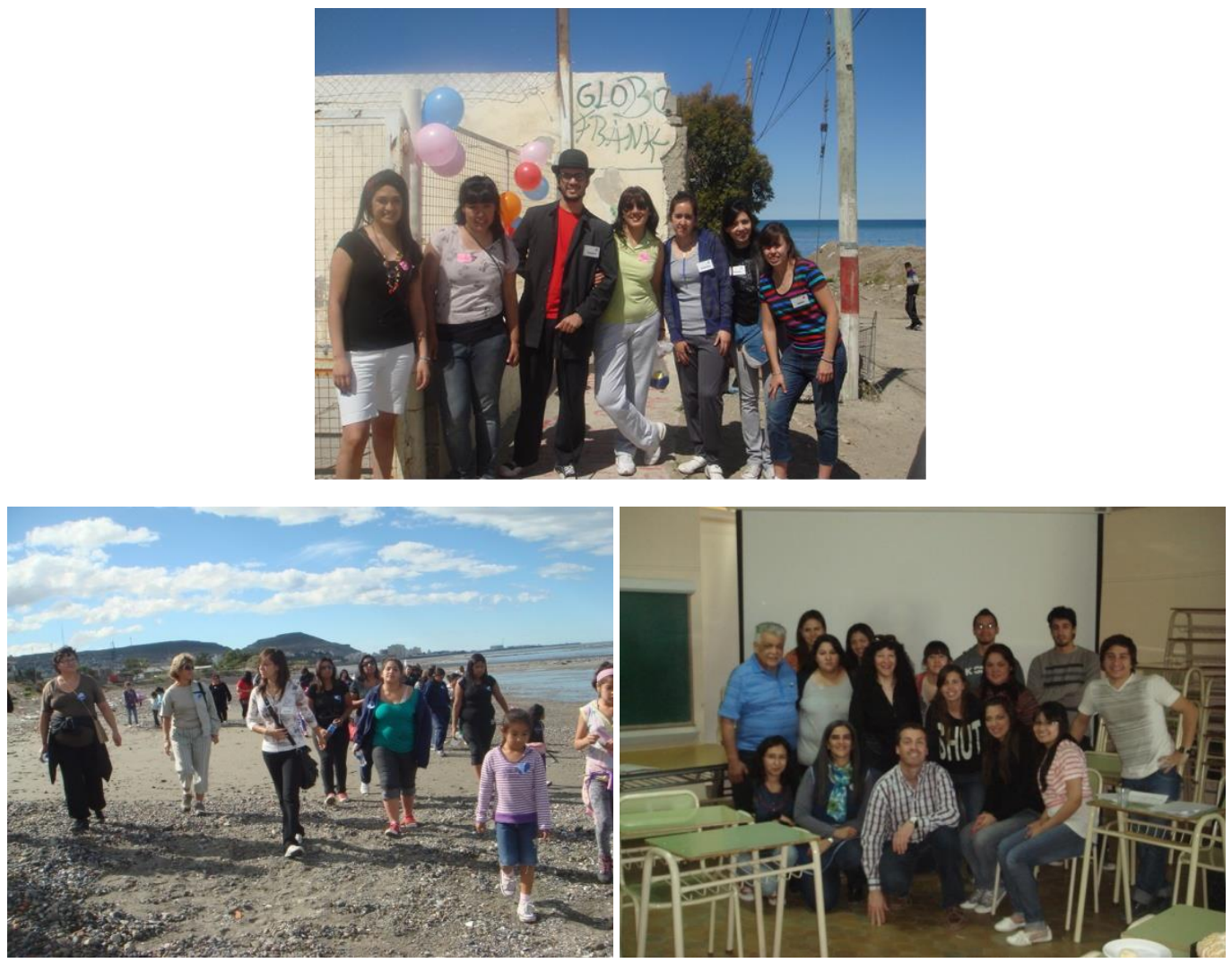

Figura 2. Tarde de playón (superior). Recuperando la memoria (inferior izquierda).

Taller educación ambiental formal (inferior derecha).

Fuente: Elaboración propia.

En cuanto al primer interrogante planteado, podría afirmarse que la totalidad de los habitantes del barrio Stella Maris son los que se exponen a las fuentes de peligro presentes $\mathrm{y}$, por lo tanto, son presumiblemente vulnerables a las mismas. Pero, si se analizan todas las dimensiones de la vulnerabilidad global, puede afirmarse que existen distintos niveles de exposición ya que no todos los habitantes van a ser igualmente vulnerables frente a las distintas fuentes de peligro identificadas. También dependerá de la capacidad de respuesta y de autoajuste que tengan los diferentes actores sociales de la comunidad barrial, lo que remite a indagar en qué medida son resistentes, o incluso resilientes frente al peligro.

Si definimos el riesgo como un concepto compuesto por dos aspectos inseparables: peligro y vulnerabilidad, debiera esperarse que en la determinación del escenario de riesgo se desarrollen con un mismo grado de atención ambos componentes. Pero dados los intereses de esta investigación, se priorizan las componentes de vulnerabilidad sobre las de peligrosidad, al entender que las primeras operan como condición interna de los sujetos, a diferencia de la peligrosidad que se define como condición externa a los mismos.

Si se analiza el concepto de vulnerabilidad, definido por Wilches-Chaux (1993) como "la incapacidad de una comunidad para 'absorber' mediante el autoajuste, los efectos de un determinado cambio en su medio ambiente, la inflexibilidad ante el cambio y la incapacidad de adaptarse al cambio" (p. 17), podría decirse que, visto el diagnóstico socioambiental del 
barrio, en el que se vierten constantemente en su costa efluentes sin tratamiento, se depositan residuos de manera indiscriminada y en el que los vecinos entrevistados manifiestan un continuo deterioro de su calidad de vida, es indudable que esta comunidad se encuentra en situación de riesgo.

Ahora bien, definidas estas condiciones a la luz de las variables conceptuales del análisis de riesgo, ¿qué rol le cabe a la educación ambiental? La pregunta en este caso obedece a la capacidad o potencialidad que tiene la educación ambiental de abordar este escenario tanto desde las componentes de la peligrosidad como desde las de la vulnerabilidad. Si el enfoque desde la educación ambiental se centrara en la peligrosidad, el análisis haría énfasis en las condiciones externas para poder comprender los impactos. Si en cambio se centrara en la vulnerabilidad, el abordaje se focalizaría en las condiciones internas de los sujetos.

\subsection{Contextos vulnerables expuestos a la peligrosidad del sitio}

El marco epistemológico de la educación ambiental por el que se ha optado se enfoca hacia el interior de la comunidad, por lo cual se priorizan algunos aspectos de la vulnerabilidad. Es por ello que, analizando los ángulos de la vulnerabilidad global, se han seleccionado dos de sus componentes: la Vulnerabilidad educativa de la comunidad y la Vulnerabilidad institucional de los decisores. La selección de la vulnerabilidad educativa se fundamenta en la consideración de que es la educación y el procesamiento y decodificación de la información de las condiciones del sitio, lo que permitirá a la comunidad reducir su propia vulnerabilidad. Del mismo modo, la elección de priorizar la vulnerabilidad institucional en la zona de estudio, tiene la intención de colaborar en la mitigación de la vulnerabilidad de las instituciones que, en palabras de Wilches-Chaux (1993:19), parecen estar diseñadas para una realidad que no se compadece con los hechos: "En general, todos sus procedimientos, parecen encaminados a impedir la respuesta estatal ágil y oportuna ante los cambios acelerados del entorno económico, político y social y del entorno ecológico".

En cuanto al segundo de los interrogantes planteados respecto al nivel de riesgo aceptable de la comunidad y de los tomadores de decisiones, la aceptabilidad del riesgo desde nuestro diagnóstico está íntimamente relacionada con la vulnerabilidad educativa de la comunidad y la vulnerabilidad institucional de los decisores, ya que si la primera no fuera vulnerable desde el punto de vista educativo, hubiera sido capaz de lograr un cambio en la tendencia de deterioro constante de su espacio costero, se hubiera organizado para lograr que las autoridades invirtieran recursos financieros en obras de saneamiento y hubieran propiciado la creación de ONG, u otra organización de la sociedad civil, que pudiera ejercer presión en los organismos gubernamentales respecto de la necesidad de mejorar la zona que habitan. Asimismo, la vulnerabilidad institucional queda evidenciada en la incapacidad de gestión efectiva de las instituciones responsables respecto de las problemáticas ambientales del barrio. Recordemos, como ejemplo, la falta de funcionamiento de una planta de tratamiento de efluentes líquidos que se instaló en el año 2001 en la zona costera del barrio, de la cual solo queda como mudo testigo una casilla abandonada.

Del mismo modo, y reforzando la teoría de la existencia de vulnerabilidad institucional, resultó muy elocuente el comentario de un responsable del Ministerio de Ambiente y Control del Desarrollo Sustentable de la provincia durante el desarrollo del Taller "Tarde de playón", cuando afirmaba que: "lamentablemente, aunque queramos, no podemos hacer demasiado, esta es una zona de sacrificio..."Una mirada desde la justicia ambiental permitiría entonces denunciar las diversas modalidades de intervención estatal, de 
emprendimientos de grupos concentrados de poder y el clasismo ambiental; a la vez posibilitaría construir prácticas y discursos alternativos para la participación en los procesos de toma de decisión pública.

\subsection{Construcción de la resiliencia desde la perspectiva de la educación ambiental}

Resultó alentador encontrar otro importante grupo de respuestas que permitieron establecer la posibilidad de construir resiliencia desde un enfoque positivo de la educación ambiental, al entender que la focalización sobre los aspectos negativos de los temas relacionados con los riesgos ensombrece los aspectos positivos (Sjöberg y Drötz-Sjoberg, 1994).Nos referimos a una mirada desde la cual se visualiza en la propia comunidad su capacidad de empoderamiento desde la cual construirla y proyectarla al entorno en el que se desenvuelven. Se valoraron las respuestas que manifestaban las ventajas de la participación ciudadana, las que visualizaron responsabilidades compartidas y las que denotaban un conocimiento de la cruda realidad pero que a su vez se acompañaban de propuestas para solucionarla. A partir de las respuestas pudieron establecerse categorizaciones desde un criterio inductivo-deductivo.

Muchas de esas respuestas rescatan la participación de la Comunidad cuando afirma “...puedo asegurarte que hay una lucha constante de los vecinos para revertir la situación”. Otras indican la responsabilidad compartida al expresar que... "La unión vecinal trabaja con estos problemas, pero esta no es la base de solución... Los vecinos tiran basura en la playa. La culpa es de todos, porque permitimos que esto pase..."; o también estas afirmaciones... "creo que la contaminación es social...tenemos una camioneta y con mi marido solemos juntar la basura de la cuadra y tirarla al basural..."

Algunas otras expresiones en este sentido de construcción de la resiliencia se centran en la ponderación positiva de las actitudes solidarias de los vecinos:

Rescato la solidaridad que hubo entre los vecinos cuando se me taparon las cañerías. ... Hemos conseguido todo gracias a los propios vecinos: ahora hay asfalto, unión vecinal, escuela, pasan los remises y el colectivo que antes no entraba al barrio. Es muy positiva la comunicación que tengo con mis vecinos lindantes.

Asimismo, otras expresan la necesidad de mayor unión entre los vecinos para resolver las problemáticas barriales:

Falta juntarnos más entre nosotros. Noto poca colaboración de las autoridades. El gas lo pusieron en cooperación entre los vecinos, la luz también entre los vecinos y los voluntarios del liceo... para poder evitar estos problemas la gente de la Manzana se debería juntar a dialogar y proponer algún tipo de solución... reunir a los vecinos y lograron acuerdo para mejorar la situación en la que vivimos.

Otros tipos de respuestas que permiten vislumbrar un tránsito hacia la construcción comunitaria de su resiliencia son las que expresan conocimiento y diagnóstico de la situación ambiental:

Trabajo del mar, pero no en esta zona, porque conozco los riesgos de comer pescado de esta playa... Antes iba a correr al mar. Ahora no por la mugre... Lo que no es del mar el mar te lo devuelve: por ejemplo, la basura, soy consciente de eso. $\Upsilon$ otras que realizan propuestas para la gestión y mejora de su espacio cotidiano.

Debería haber un ente que regularice los desechos de gasoil, de las pesqueras, industriales, debe haber una cisterna que filtre los desechos... Hace falta un plan de desarrollo municipal... Es necesario ampliar el emisario y que desagüe 500 metros adentro. Ampliaron el entubado para que puedan emitirse mayor cantidad de efluentes. Los desechos cloacales no se pueden revertir, esto viene desde hace años, esto 
se soluciona con plantas de tratamiento, el entubado no sirve con marea baja y viento de mar se nos viene el olor. Las plantas de tratamiento serían lo mejor. Y arrojar los residuos en un sector que se pueda controlar... Hay que hacer plantas de tratamiento y controlar a la gente que tira cosas, poner a alguien para que vigile el lugar.

Por último, se identificaron las respuestas que evidencian una apuesta al cambio de actitudes, la toma de conciencia y la educación. Muchas de ellas centran las responsabilidades en los tomadores de decisiones, al considerar que son ellos los que a partir de las acciones de gestión local pueden cambiar las realidades ambientales en el territorio ya que están vinculadas a decisiones políticas en la asignación y reparto de recursos económicos, humanos y financieros:

\footnotetext{
Hay que hacer bien las cosas y que haya continuidad en las acciones, aunque cambien los gobiernos. Deben reunir a los del barrio y presentar proyectos para limpiar la costa... En vez de hacer campañas políticas hubieran invertido para limpiar el barrio. Las autoridades actúan mal, no dan respuestas. Todo lo que te digo molesta a las autoridades que no hacen nada y solo van donde hay plata. No le dan importancia, nada hacen para recuperar la costa.
}

Entre las que refieren a los cambios de actitudes, conciencia y necesidad de educación se destacan las siguientes:

\begin{abstract}
... falta concientización, hay que estar controlando, somos así, si no te están pinchando no lo hacemos. Falla la justicia, faltan sanciones... Este daño ambiental se podría haber evitado con la concientización de la gente porque la costa es de todos... No se les enseña en el colegio a los chicos sobre educación ambiental pero la educación depende en gran medida de una buena base familiar... No me gusta tirar la basura al mar, no saco la basura hasta media hora antes de que pase el camión y genero una bolsa por semana, eso lo dejo a conciencia de cada uno... Nuestra familia es muy consciente de este problema y tratamos de conservar la limpieza, no sé por qué hay tanta basura.
\end{abstract}

Asimismo, hubo propuestas "a corazón abierto" que implican el compromiso de la comunidad entre las que destacamos:

\begin{abstract}
No los llamaría conflictos, sino que haría una pregunta, ¿̨cómo ayudamos? Soluciones: juntar gente para limpiar la costa. Siempre hay chicos gente joven que ayuda... Se podría solucionar los conflictos ambientales con charlas a los vecinos, reuniones con vecinos, hacer público por $T V$, carteles, información en las escuelas... Me gusta que se haya formado el voluntariado, con el objetivo de mejorar la costa y si llegan a necesitar algo estoy dispuesto para ayudar.
\end{abstract}

\title{
4. Conclusiones
}

\subsection{Algunos cambios producidos}

Si bien la tarea de causar un cambio de actitudes frente al medio es lenta y debe valorarse a medio y largo plazo, existen evidencias alentadoras que, creemos, han conducido a la disminución de la vulnerabilidad educativa frente al peligro de contaminación, especialmente por haber conseguido la movilización, aunque aún incipiente, de la comunidad educativa del barrio que se ha involucrado activamente en los talleres y acciones participativas propuestas. Lo novedoso de la justicia ambiental no es lograr una elevada conciencia ambiental, sino potenciar las posibilidades de un cambio social y ambiental a través de procesos de redefinición, reinvención y construcción de discursos y prácticas políticas y culturales. Ello implica la articulación de las nociones de justicia ambiental y de discriminación (segregación) ambiental, y la construcción de nuevas formas de organización política de base (Di Chiro, 1998). 
En cuanto a la disminución de la vulnerabilidad institucional, tan común en escenarios como el que nos ocupa, donde los actores sociales y sus sufrimientos no son prioritarios en las agendas de gobierno, consideramos que la participación de los referentes institucionales en acciones de gestión ambiental local nos indica testimonios concretos en esa línea. Citamos como ejemplos la asistencia al Congreso de Vecinalismo 2013, al que llevaron como propuesta para su tratamiento y debate el caso de la necesidad de recuperar el Parque del 99, como asimismo sus testimonios expresados en los medios de comunicación local dando cuenta de las actividades de educación ambiental realizadas y los talleres de organización conjunta que se organizaron en el barrio durante estos años. De algún modo estas acciones van iniciando un camino tendiente a cuestionar el desinterés, burocratización o negligencia, directos indicadores de esta vulnerabilidad institucional.

Por otra parte, concretaremos los principales cambios que se han generado en estos años a partir del desarrollo del programa. Consideramos que uno de los más relevantes en el territorio fueron las acciones de mejora y revalorización de la zona costera que se plantean en los senderos interpretativos y que se han dado a conocer tanto a las autoridades educativas del barrio como a dos de los representantes de la gestión política ambiental. Asimismo, el haber decidido la construcción del edificio donde funcionará la delegación del laboratorio de aguas dependiente de la Subsecretaría de Ambiente Municipal sobre la costa y aledaño a la planta de tratamiento de efluentes, hoy inhabilitada por mal funcionamiento, es una señal de la intención política de aproximarse al problema de contaminación de este sector costero. Esto último, si bien no es atribuible al programa de educación ambiental, ha sido señalado como un logro por parte de los directivos de las escuelas quienes afirman que, de no haberse visibilizado la problemática a partir de las acciones de educación ambiental desarrolladas en el barrio, tal vez no se hubiera decidido su construcción definitiva. Esta medida del área ambiental municipal confirma además que el sitio elegido como escenario de este trabajo es un espacio considerado prioritario para la gestión política entre las acciones de saneamiento en la zona costera.

En cuanto a los cambios en las instituciones hemos logrado sumar las propuestas de contenidos curriculares específicos de educación ambiental y temáticas costeras, que se han desarrollado tanto en el nivel inicial como primario y secundario. Esas instituciones cedieron horas curriculares, que originalmente estaban destinadas a otras disciplinas, para el tratamiento de las temáticas y actividades costeras que propusimos desde los voluntariados. Por su parte, los alumnos del Colegio Secundario participaron de actividades extracurriculares no obligatorias, como fue el caso de la salida para el avistaje de aves o su participación dentro del certamen de recuperación de la memoria ambiental que propusimos. Este es un primer paso, pero de suma importancia en la búsqueda de un cambio de actitudes de los jóvenes del barrio y ha sido señalado positivamente por los directivos de la escuela.

Los cambios favorables producidos en los alumnos y voluntarios han sido reflejados en sus encuestas de opinión y lo más destacado ha sido la "utilidad en su formación y la posibilidad de replicar estas experiencias de educación ambiental” en otros espacios costeros, lo que abre nuevas perspectivas de investigación.

\subsection{Principales fortalezas}

Entre las fortalezas más destacables del proceso de investigación-acción desarrollado a lo largo de los tres años que duró la intervención podemos destacar el reconocimiento del 
trabajo en red desde las instituciones barriales. Todos ellos se conocen y se reconocen en un mismo nivel de acción. Los representantes institucionales llevan muchos años en el barrio, por lo cual son testimonios vivientes de los procesos sociales que se han venido consolidando. Esa fue desde un comienzo una ventaja en el momento de comenzar la intervención ya que, de no encontrar una comunidad previamente organizada, la tarea no hubiera sido posible o no hubiera producido frutos.

Resaltamos ese compromiso al haber sido testigos de la concurrencia de la gran parte de los responsables institucionales a diferentes eventos tales como reuniones organizativas, convocatorias específicas ante dificultades o acontecimientos puntuales que hacen a las necesidades del barrio.

Una fortaleza destacada por los alumnos universitarios fue el constatar que las instituciones nos habían incorporado como un actor más del barrio, tal fue el caso de la invitación a formar parte de reuniones organizadas con el equipo político municipal realizada en el mes de abril de 2013 en la Unión vecinal. Han destacado además la posibilidad de haber fortalecido los lazos entre las instituciones barriales y la universidad.

Haber encontrado una "punta de ovillo" a la problemática histórica de la contaminación del sector, como fue la idea de recuperar la memoria ambiental de la playa, también ha sido señalado como un punto fuerte del Programa. Fue muy importante para los voluntarios el haber reunido fotos antiguas aportadas por los vecinos y haberlas exhibido púbicamente en las escuelas del barrio. Del mismo modo, las acciones para intentar recuperar el Parque del 99 constituyeron otra de las estrategias más palpables para avanzar en la recuperación de la calidad ambiental de la playa.

\subsection{Puntos débiles}

Uno de los aspectos que aún no hemos podido revertir es la naturalización de la problemática ambiental existente, en especial en los niños. Este sería uno de los principales desafíos a enfrentar para promover procesos sostenidos de reconocimiento, de situaciones de privación de derechos que movilicen reclamos de justicia ambiental. Las evaluaciones de esta experiencia de trabajo en el territorio realizadas por docentes y alumnos universitarios dan cuenta del sentimiento extraño que causó el ver a los niños caminando por encima de un efluente que descarga en la playa sin mayor inconveniente. Lo mismo ocurrió con las acciones de las personas y el doble discurso: por un lado, querer una playa limpia y por otro seguir arrojando basura.

Gran parte de los actores con los que hemos trabajado consideran que una de las mayores problemáticas con la que nos enfrentamos es el nivel de riesgo de contaminación con el que se ha acostumbrado a vivir la población costera. Estos aspectos perceptivos y culturales deberían ser trabajados a través de proyectos que se lleven a cabo en todas las escuelas de la ciudad, no solo en las del barrio.

El programa tampoco ha podido encontrar hasta el momento el modo de involucrar a los actores privados en una puesta en valor de este espacio. Consideramos que ese es un punto importante para seguir trabajando. Quizá la figura de la responsabilidad social empresarial pueda ser una medida que inicie este camino de mitigación del daño ambiental que ha causado, entre otros aspectos, la actividad industrial en el barrio.

Algo similar ocurre con la gestión política. Salvo escasas excepciones estos actores no han considerado la posibilidad de trabajo conjunto en el territorio con el voluntariado 
universitario en acciones de educación ambiental. Sin ir más lejos, en dos programas actuales se está instalando desde el discurso político la temática de la clausura del actual basural a cielo abierto por lo que, manifiestan, se van a iniciar acciones y campañas de concienciación y de educación ambiental para separar residuos en los domicilios. En la misma tónica, varios de los integrantes del voluntariado opinaron en sus evaluaciones que las cuestiones asociadas a las decisiones y propuestas de mejora en la gestión del barrio escaparían a las acciones del voluntariado.

Es por ello que las acciones de educación ambiental desplegadas han sido necesarias, pero aún no son suficientes para modificar las actitudes y conductas ciudadanas en la relación de la comunidad y su ambiente cotidiano. Ello constituye entonces uno de los desafíos más importantes que nos motivan a seguir trabajando de manera continua con la comunidad en la que ya hemos intervenido. De ese modo se irán visibilizándolas condiciones de privación del derecho al ambiente sano que atraviesan la vida comunitaria y que requieren de la construcción de un sujeto popular (barrial) que reclame, mediante el despliegue de poder creativo, nuevas formas de reconocimiento que constituyan condiciones para la justicia ambiental.

\section{Referencias}

Al-Balushia, S. M. y Al-Aamri, S. S. (2014). The effect of environmental science projects on students' environmental knowledge and science attitudes. International Research in Geographical and Environmental Education, 23, 213-227. https://doi.org/10.1080/10382046.2014.927167

Barragán-Muñoz, J. M. (2003). Medio ambiente y desarrollo en áreas litorales: Introducción a la planificación y gestión integradas. Cádiz: Servicio de Publicaciones de la Universidad de Cádiz.

Bunge, M. (1989). La investigación científica. Barcelona. Editorial Ariel.

Carvalho, I. (2004). Educaçao ambiental: A formaçao do sujeito ecológico. Sao Paulo: Ed. Cortez.

Carrizo, C. (2012). La soberanía popular y la justicia ambiental. En C. Carrizo y M. Berger (Comps.), Justicia ambiental y creatividad democrática. Córdoba: Alción Editora.

Cohen, L., Manion, L. y Morrison, K. (2005). Research methods in education. Londres: Taylor \& Francis.

Di Chiro, G. (1998). La justicia ambiental y la justicia social en Estados Unidos: La naturaleza como comunidad. En M. Goldman (Ed.), Privatizing nature. Political struggles for the global commons. Londres: Pluto Press y Transnational Institute.

Freire, P. (1970). Pedagogia do oprimido. Rio de Janeiro: Paz e Terra.

George P. W. A. (1972). L'environnement. Population, 27, 555-556. https://doi.org/10.2307/1529446

Hernández-Sampieri, R., Fernández, C. y Baptista, P. (1991). Metodología de la investigación. Bogotá: Presencia.

Latapí, P. (1995). Educación y justicia: Términos de una paradoja. Washington, DC: OEA.

Liarakou, G., Kostelou, E. y Gavrilakis, C. (2011). Environmental volunteers: Factors influencing their involvement in environmental action. Environmental Education Research, 17, 651-673. https://doi.org/10.1080/13504622.2011.572159 
Mateos, A., Bejarano, M. y Moreno, D. (2014). Los cuentos y los juegos de simulación para trabajar la justicia social en el ámbito de las ciencias en las primeras edades. Revista Internacional de Educación para la Justicia Social, 3, 97-119.

Maulucci, M. (2012). Social justice research in science education: Methodologies, positioning, and implications for future research. En B. J. Fraser (Dir.), Second international handbook of science education (pp. 583-594). Nueva York, NY: Springer. https://doi.org/10.1007/978-1-40209041-7_40

Paige, K. y Hardy, G. (2014). Socio-scientific issues: A transdisciplinary approach for engaging pre-service teachers in science and mathematics education. Revista Internacional de Educación para la Justicia Social, 3, 17-36.

Prades-López A. y González-Reyes F. (1999). La percepción social del riesgo: Algo más que discrepancia experto/público. Revista Nucleus, 26, 3-12.

Raimondo A. (2008). Necesidad de la educación ambiental para el abordaje de problemáticas en un espacio de complejidad: La costa de Comodoro Rivadavia - Chubut-Patagonia Argentina. En J. Gutiérrez y L. Cano (Eds.), Investigaciones en la década de la educación para el desarrollo sostenible (pp. 225-243). Madrid: Organismo Autónomo de Parques Nacionales. https://doi.org/10.1080/10382046.2016.1217077

Raimondo A. (2010). Propuesta para una definición de la franja costera, usos y actividades en la costa de C. Rivadavia (Chubut, Patagonia Argentina). IGEOPAT, 9, 66-100.

Raimondo A. M., Gutiérrez-Pérez, J. y Perales-Palacios F. J. (2012). Formación y educación ambiental. Una experiencia integradora de aprendizaje basado en los problemas del territorio. Profesorado. Revista de Currículum y Formación de Profesorado, 16(2), 239-256.

Raimondo, A., Monti, A., Perales-Palacios, F. J. y Gutiérrez-Pérez, J. (2017). Evaluation of an action-research project by university environmental volunteers. International Research in Geographical and Environmental Education, 26, 54-70.

Raimondo, A. M. (2014). Aportes de la educación ambiental a la reducción de la vulnerabilidad educativa del barrio Stella Maris (Chubut, Argentina): Diseño, desarrollo y evaluación de un programa estratégico mediante investigación-acción participativa (Tesis doctoral) Universidad de Granada, España.

Rodríguez, A. J. (1997). The dangerous discourse of invisibility: A critique of the national research council's national science education standards. Journal of Research in Science Teaching, 34, 19-37. https://doi.org/10.1002/(SICI)1098-2736(199701)34:1<19::AID-TEA3>3.0.CO;2$\mathrm{R}$

Sauvé, L. (2006). La educación ambiental y la globalización: Desafíos curriculares y pedagógicos. Revista Iberoamericana de Educación Ambiental, 41, 83-102.

Schlosberg, D: (2004). Reconceiving environmental justice: Global movements and political theories. Environmental Politics, https://doi.org/10.1080/0964401042000229025

Sjöberg, L. y Drotz-Sjöberg, B. M. (1994). Risk perception of nuclear waste: Experts and the public. Estocolmo: Centre of Risk Research, Stockholm School of Economics.

Sorensen, J. C., Mc Creary, S. T. y Brandani, A. (1992). Costas: arreglos institucionales para manejar ambientes $y$ recursos costeros. Kingston, RI: United State Agency for Internacional Development.

Suárez-Pasos, M. (2002). Algunas reflexiones sobre la investigación-acción colaboradora en la educación. Revista Electrónica de Enseñanza de las Ciencias, 1, 40-56. 
Vázquez, L. (2015). De la ocupación a la legislación. Marco legal sobre el suelo urbano en Comodoro Rivadavia (1901-1996). Identidades, 9, 100-112.

Wilches-Chaux, G. (1993). La vulnerabilidad global. En A. Maskrey (Comp.), Los desastres no son naturales. Recuperado de http://www.lared.org/public/libros/1993/ldnsn/LosDesastresNoSonNaturales-1.o.o.pdf

\section{Breve CV de los autores}

\section{Ana M. Raimondo}

Profesora y Licenciada en Geografía por la Universidad Nacional de la Patagonia San Juan Bosco (UNPSJB). Doctora en Educación Ambiental por la Universidad de Granada. Profesora Titular Exclusiva de la Cátedra de Educación Ambiental. Facultad de Humanidades y Ciencia Sociales (UNPSJB). Miembro titular del Grupo de Estudios Geográficos en espacios Costeros (GECOS) perteneciente al Instituto de Investigaciones Geográficas de la Patagonia (IGEOPAT). Directora del proyecto de Investigación: Afianzando la Educación Ambiental y la Intervención Comunitaria en Espacios Litorales de Comodoro Rivadavia. Coordinadora de la Maestría en Geografía de los Espacios Litorales (UNPSJB). ORCID ID: OOOO-OO01-7434-0815. Email: ana.raimondo@gmail.com

\section{F. Javier Perales-Palacios}

Doctor en Ciencias Físicas por la Universidad de Granada (España), Catedrático de Universidad, trabaja en la Facultad de Ciencias de la Educación, habiendo impartido diversas asignaturas relacionadas especialmente con la Física, la Educación Ambiental y la Didáctica de las Ciencias, así como másteres y cursos de doctorado en diversos países. En cuanto a la actividad investigadora, ha participado como colaborador o investigador principal en 21 proyectos, tanto a nivel nacional como internacional y ha dirigido 18 tesis doctorales, siendo autor de 139 artículos en revistas nacionales e internacionales y publicado o editado 57libros, capítulos o monografías. Es responsable del grupo de investigación Didáctica de las Ciencias Experimentales y de la Sostenibilidad. ORCID ID: oooo-0002-6112-2779.Email: fperales@ugr.es

\section{José Gutiérrez-Pérez}

Profesor de Métodos de Investigación y Diagnóstico en Educación en el Departamento MIDE de la Universidad de Granada. Dirige el grupo de Investigación Evaluación en Educación Ambiental, Social e Institucional (HUM-890). Su línea de investigación principal se centra en el tópico de la evaluación de la calidad de los programas, centros y recursos de Educación Ambiental, sobre el que ha publicado artículos, libros y contribuciones en encuentros científicos especializados. ORCID ID: OOOO-0003-42119694. Email: jguti@ugr.es

\section{Susana Vidoz}

Doctoranda en Ciencias Sociales (Universidad de Buenos Aires). Magister en Políticas Sociales (Universidad de Buenos Aires). Licenciada en Ciencia Política (Universidad de Buenos Aires. Profesora Adjunta de la cátedra Teoría Política y Social I en la carrera de Ciencia Política (online) y de la cátedra de Gestión Social y Política en la carrera de 
Gestión Ambiental, de la Facultad de Humanidades y Ciencias Sociales (Universidad de la Patagonia San Juan Bosco). ORCID ID: O000-0003-4113-3232. Email: susanavidoz@gmail.com 